\title{
High Expression of the "A Disintegrin And Metalloprotease" 19 (ADAM19), a Sheddase for TNF- $\alpha$ in the Mucosa of Patients with Inflammatory Bowel Diseases
}

\author{
Eleonora Franzè, PhD, ${ }^{*}$ Roberta Caruso, MD, PhD, ${ }^{*}$ Carmine Stolfi, PhD, ${ }^{*}$ Massimiliano Sarra, PhD, ${ }^{*}$ \\ Maria Laura Cupi, PhD,* Marta Ascolani, MD, ${ }^{*}$ Silvia Sedda, MD,* Claudia Antenucci, MD,* \\ Alessandra Ruffa, MD, ${ }^{*}$ Flavio Caprioli, MD, PhD, ${ }^{+}$Thomas T. MacDonald, PhD, ${ }^{\neq}$Francesco Pallone, MD, ${ }^{*}$ \\ and Giovanni Monteleone, MD, PhD*
}

\begin{abstract}
Background: Tumor necrosis factor $\alpha$ (TNF- $\alpha$ ) plays a major role in the tissue-damaging immune response in inflammatory bowel diseases (IBDs). The tissue concentration of TNF- $\alpha$ is related to the activity of "A Disintegrin And Metalloprotease" (ADAMs), enzymes that process membranebound TNF- $\alpha$ and liberate the TNF- $\alpha$ trimer into the extracellular environment. Although IBD-related inflammation is associated with high ADAM17 levels, the contribution of other members of the ADAMs family is not known. In this study, we characterized the expression of other TNF- $\alpha$ convertases (i.e., ADAM9, ADAM10, and ADAM19) in IBD.
\end{abstract}

Methods: Normal and IBD biopsies were examined for the content of ADAMs by real-time polymerase chain reaction, Western blotting and immunohistochemistry. ADAM19 was also analyzed in intestinal epithelial cells and normal colonic explants stimulated with inflammatory cytokines and in ex vivo biopsies taken from IBD patients before and after a successful infliximab treatment.

Results: ADAM19 RNA transcripts and protein were upregulated in patients with ulcerative colitis and, to a lesser extent, in patients with Crohn's disease compared with normal controls. In contrast, ADAM9 and ADAM10 expression did not differ between patients with IBD and controls. Immunohistochemical analysis showed that epithelial cells were the major source of ADAM19 in IBD. ADAM19 expression was increased in colonic epithelial cell lines and normal colonic explants by TNF- $\alpha$, interleukin 21 and interleukin 6 , and was downregulated in IBD tissue by infliximab.

Conclusions: These findings suggest the existence of a positive feedback mechanism involving cytokines and ADAM19 that can amplify cytokine production in IBD.

(Inflamm Bowel Dis 2013;19:501-511)

Key Words: ADAM19, TNF- $\alpha$, inflammatory bowel disease, colitis

$\mathrm{C}$ rohn's disease (CD) and ulcerative colitis (UC), the main forms of inflammatory bowel diseases (IBDs) in humans, are chronic relapsing disorders of the intestine. ${ }^{1}$ The cause of

Supplemental digital content is available for this article. Direct URL citations appear in the printed text and are provided in the HTML and PDF versions of this article on the journal's Web site (www.ibdjournal.org).

Received for publication May 31, 2012; Accepted June 29, 2012.

From the *Department of Internal Medicine, University of Rome "Tor Vergata", Rome, Italy; "Unit of Gastroenterology, Fondazione IRCCS Cà Granda Ospedale Maggiore Policlinico, Department of Pathophysiology and Transplantation, University of Milan, Milan, Italy; and Institute of Cell and Molecular Science Centre for Infectious Diseases, Barts and the London School of Medicine and Dentistry, London, United Kingdom.

Supported by "Fondazione Umberto Di Mario ONLUS" (Rome, Italy), Giuliani S.p.a. (Milan, Italy), funding for the IPODD consortium under Grant Agreement 202020 of the Seventh Research Framework Programme of the European Union.

The authors have no conflicts of interest to disclose.

Reprints: Giovanni Monteleone, $\mathrm{MD}, \mathrm{PhD}$, Dipartimento di Medicina Interna, Università Tor Vergata, Via Montpellier, 1, 00133 Rome, Italy (e-mail: gi.monteleone@, med.uniroma2.it).

Copyright (C) 2013 Crohn's \& Colitis Foundation of America, Inc.

DOI 10.1097/MIB.0b013e31828028e 8

Published online 6 February 2013.
IBD is unknown, but in recent years it has become evident that IBD is probably caused by excessive immune reactivity within the intestinal wall directed against luminal bacterial antigens., ${ }^{2,3}$ The mechanisms that contribute to amplify and maintain the destructive mucosal inflammation are not yet fully understood, but there is evidence that proinflammatory cytokines are involved in the tissue-damaging inflammatory reaction. ${ }^{1-3}$ One such cytokine is tumor necrosis factor $\alpha$ (TNF- $\alpha$ ), mostly produced by macrophages, which can activate several inflammatory pathways in both immune and nonimmune cells. ${ }^{4-6}$ Blockade of TNF- $\alpha$ with neutralizing antibodies (i.e., infliximab [IFX] or adalimumab) can induce and maintain remission in approximately $50 \%$ of patients with $\mathrm{IBD}^{7-11}$ However, not all patients respond to anti-TNF, and response wanes with time. ${ }^{12,13}$ Moreover, the blockade of TNF- $\alpha$ is associated with infections and/or new immune-mediated pathologies, ${ }^{8,14-17}$ suggesting the need for novel strategies, particularly in patients who do not respond to or cannot receive TNF blockers.

Very little is known about factor(s) involved in the regulation of TNF- $\alpha$ in the inflamed gut of patients with IBD. TNF- $\alpha$ production can be positively regulated at transcriptional 
level by pathogen-associated molecular patterns, such as lipopolysaccharide or bacterial peptidoglycan derivatives, which activate nuclear factor $\kappa \mathrm{B}$ and mitogen-activated protein kinases. ${ }^{4,18} \mathrm{TNF}-\alpha$ is synthesized as a trimeric type 2 membrane-anchored precursor referred to as pro-TNF- $\alpha .{ }^{19}$ The mature form of TNF- $\alpha$ is released from the cell by the action of proteolytic enzymes. ${ }^{20}$ The TNF- $\alpha$ covertase "A Disintegrin And Metalloprotease (MMP) domain" (ADAM) 17 , also termed TACE (TNF- $\alpha$ converting enzyme), is critical for processing TNF- $\alpha{ }^{21}$ ADAM17 is upregulated in IBD and in experimental models of colitis, and in the latter, intraperitoneal administration of a TACE inhibitor ameliorates colonic damage and inflammation. ${ }^{22,23}$ Moreover, in vitro studies have shown that ADAM9, ADAM10, and ADAM19 can also cleave transmembrane-bound TNF- $\alpha{ }^{24}$ This study was therefore designed to explore the expression and regulation of these other ADAMs in IBD.

\section{MATERIALS AND METHODS}

\section{Patients and Samples}

Mucosal biopsies were taken from the inflamed areas of 12 patients with colonic $\mathrm{CD}, 8$ patients with ileocolonic $\mathrm{CD}, 3$ patients with ileal $\mathrm{CD}$, and 21 patients with UC undergoing colonoscopy for clinically active disease at the Gastrointestinal Unit of either Tor Vergata University (Rome) or Fondazione IRCCS Cà Granda, Ospedale Maggiore Policlinico in Milan. Moreover, paired biopsies were taken from the inflamed and uninflamed mucosa of 4 patients with CD (1 colonic and 3 ileocolonic) and 8 patients with UC. In 10 patients ( 3 colonic $\mathrm{CD}, 3$ ileocolonic $\mathrm{CD}$, and $4 \mathrm{UC}$ ), who were taking no drug, biopsies were collected at the time of initial diagnosis of disease; 19 patients ( 2 colonic CD, 3 ileocolic CD, 2 ileal $\mathrm{CD}$, and $12 \mathrm{UC}$ ) were receiving mesalamine and underwent endoscopy for a clinical relapse of the disease; 11 patients $(2$ colonic $\mathrm{CD}$, 2 ileocolonic $\mathrm{CD}$, and $7 \mathrm{UC}$ ) were taking steroids; and 7 patients (4 colonic $\mathrm{CD}, 1$ ileal $\mathrm{CD}$, and $2 \mathrm{UC}$ ) were receiving immunosuppressive drugs. Five steroid-dependent and/or steroid-resistant patients (2 colonic CD, 1 ileocolonic $\mathrm{CD}$, and $2 \mathrm{UC}$ ) were treated with IFX. Each patient received a total of 3 IFX infusions $(5 \mathrm{mg} / \mathrm{kg})$ at weeks 0,2 , and 6 . Six colonic biopsies were collected during a flexible sigmoidoscopy from the sigmoid colon before the first and after the third IFX infusion. All these patients were defined "responders" because a complete mucosal healing was documented after the last IFX infusion. Using Simple Endoscopic Score for Crohn's disease and Mayo endoscopic subscore, the endoscopic activity was evaluated. ${ }^{25,26}$ Two patients (1 CD and 1 UC) were receiving mesalamine and azathioprine, 2 patients with $\mathrm{CD}$ were treated with mesalamine only because they were intolerant to immunosuppressors, and 1 patient with UC was receiving mesalamine, azathioprine, and steroids. All the concomitant therapies were maintained during the IFX treatment.

Additionally, surgical specimens were taken from 8 patients with colonic $\mathrm{CD}, 8$ patients with UC undergoing surgery for a chronic active disease poorly responsive to medical treatment, and 3 patients with ileal CD undergoing surgery as a result of stricturing disease. In 3 of 8 patients with UC, surgical specimens were taken from both involved and uninvolved mucosa.

Controls included biopsies taken from unaffected colonic mucosa of 18 subjects and from unaffected ileal mucosa of 6 subjects undergoing colonoscopy for colorectal cancer screening. Additional controls were mucosal specimens taken from macroscopically and microscopically unaffected colonic areas of 8 patients and ileal areas of 3 patients who underwent surgery for colon cancer. Informed consent was obtained from all patients, and the local ethics committees approved the study protocol.

\section{Cell Culture}

All the reagents were from Sigma-Aldrich (Milan, Italy) unless specified. The human colorectal cancer cell line, DLD-1, was maintained in RPMI 1640 medium supplemented with $10 \%$ fetal bovine serum, penicillin (P) $(100 \mathrm{U} / \mathrm{mL})$, streptomycin $(\mathrm{S})$ $(100 \mu \mathrm{g} / \mathrm{mL})$, and gentamicin $(\mathrm{G})(50 \mu \mathrm{g} / \mathrm{mL})$ (all from Lonza, Verviers, Belgium) in 6-well culture dishes. After reaching $60 \%$ to $70 \%$ confluence, cells were starved for 12 hours. Nonadherent cells were then removed, and fresh medium containing $0.05 \%$ bovine serum albumin was added to each well. To evaluate whether inflammatory stimuli could modulate ADAM19 expression, cells were either left unstimulated or stimulated with TNF- $\alpha$, interleukin (IL)-6 (both used at a concentration of $20 \mathrm{ng} / \mathrm{mL}$; R\&D Systems, Minneapolis, MN), and IL-21 (100 ng/mL; Prosci, Inc., Poway, CA) for 3 to 48 hours. To evaluate whether IFX directly controls ADAM19 expression, DLD-1 cells were cultured in the presence of $50 \mu \mathrm{g} / \mathrm{mL}$ IFX or control human IgG (R\&D Systems) for 6 hours and then analyzed by real-time PCR.

\section{Flow Cytometry}

To assess cell viability, DLD-1 cells were cultured with $50 \mu \mathrm{g} / \mathrm{mL}$ IFX or control IgG for 6 hours. Cells were then collected, washed twice in phosphate-buffered saline $1 \mathrm{X}$ and stained with fluorescein isothiocyanate-annexin V (AV) (1:100 final dilution) according to the manufacturer's instructions (Becton Dickinson, Milan, Italy), and incubated with $5 \mu \mathrm{g} / \mathrm{mL}$ propidium iodide (PI) for 30 minutes at $4^{\circ} \mathrm{C}$. Fluorescence was measured using FL-1 and FL-2 channels of FACSCalibur (Becton Dickinson, Milan, Italy) using CellQuest Pro software (Becton Dickinson). $\mathrm{AV}-/ \mathrm{PI}-$ cells were considered as viable cells.

\section{Ex Vivo Organ Cultures}

Freshly obtained intestinal mucosal samples were cultured as described elsewhere. ${ }^{27}$ Briefly, samples were placed on iron grids with the mucosal face upward in the central well of an organ culture dish containing AQIX medium (Aqix Ltd., London, UK) supplemented with $1 \%$ L-glutamine (Lonza) and $\mathrm{P} / \mathrm{S} / \mathrm{G}$. Colonic biopsies were taken from 4 normal controls (NC) and incubated in the presence or absence of TNF- $\alpha$, IL-6 (both used at a concentration of $20 \mathrm{ng} / \mathrm{mL})$, or IL-21 (100 ng/mL), whereas CD biopsies taken from 4 patients were incubated with IFX or control human IgG (both at a concentration of $50 \mu \mathrm{g} / \mathrm{mL}$ ). Dishes were then placed in a tight container with $95 \% \mathrm{O}_{2}$, with $5 \% \mathrm{CO}_{2}$ at $37^{\circ} \mathrm{C}$, at 1 bar. After 
24 hours of culture, mucosal samples were homogenized with Tissue Lyser II (Qiagen S.r.l., Milan, Italy) in TRIzol reagent (Invitrogen, Milan, Italy), and total RNA was extracted according to the manufacturer's instructions (Invitrogen, Milan, Italy). In parallel, culture supernatants were collected and stored at $-80^{\circ} \mathrm{C}$ until assessed for ADAM19 expression by Western blotting.

\section{RNA Extraction, Complementary DNA Preparation, and Real-Time PCR}

Total RNA was extracted using TRIzol reagent. A constant amount of RNA ( $1 \mu \mathrm{g} / \mathrm{sample})$ was retrotranscribed into complementary DNA (cDNA), and then, $1 \mu \mathrm{L}$ of cDNA per sample was amplified by real-time PCR. ADAM17, ADAM9, ADAM10, and ADAM19 were evaluated using commercially available TaqMan probe (Applied Biosystems, Foster City, CA). $\beta$-Actin (forward primer, 5'-AAGATGACCCAGATCATGTTTGAGACC-3'; and reverse primer, 5'-AGCCAGTCCAGACGCAGGAT-3') was used as internal control gene. The cDNA was amplified using the following conditions: denaturation for 1 minute at $95^{\circ} \mathrm{C}$, annealing for 30 seconds at $60^{\circ} \mathrm{C}$ followed by 30 seconds of extension at $72^{\circ} \mathrm{C}$. ADAM17, ADAM9, ADAM10, and ADAM19 RNA expression was calculated relative to the housekeeping $\beta$-actin gene on the base of the $\Delta \Delta \mathrm{Ct}$ algorithm.

\section{Total Protein Extraction and Western Blotting}

Colonic samples of $9 \mathrm{NC}, 9$ patients with $\mathrm{UC}$, and 9 patients with $\mathrm{CD}$, and DLD-1 cells were lysed on ice in buffer containing
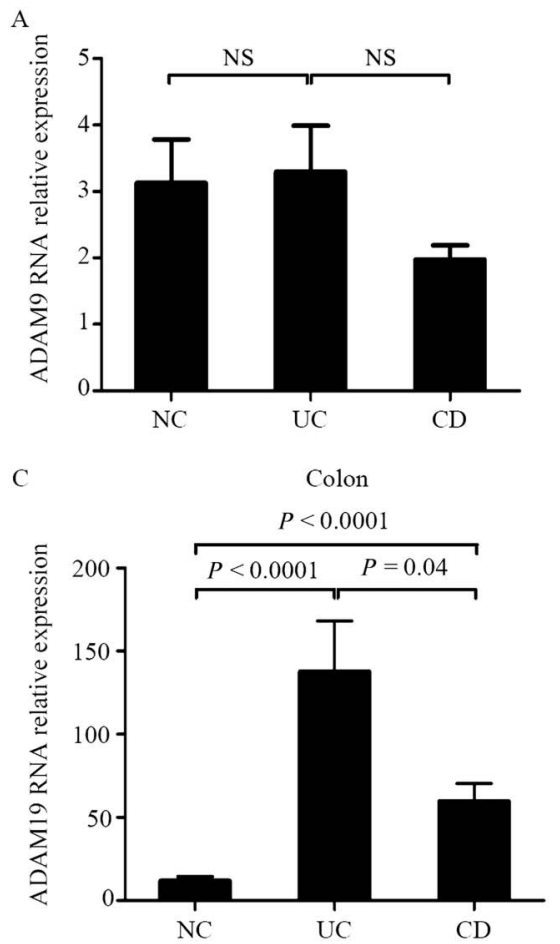

FIGURE 1. ADAM19 transcripts are increased in IBD. A-C, ADAM9 (A), ADAM10 (B), and ADAM19 (C) RNA expression was evaluated in colonic biopsies taken from 18 normal controls (NC), 25 patients with UC, and 19 patients with CD by real-time PCR, and levels were normalized to $\beta$-actin. Data indicate mean \pm SEM of all samples. D, ADAM19 RNA expression was evaluated in ileal biopsies taken from 6 NC and 4 patients with CD by real-time PCR. Data indicate mean \pm SEM of all samples. NS, not significant.

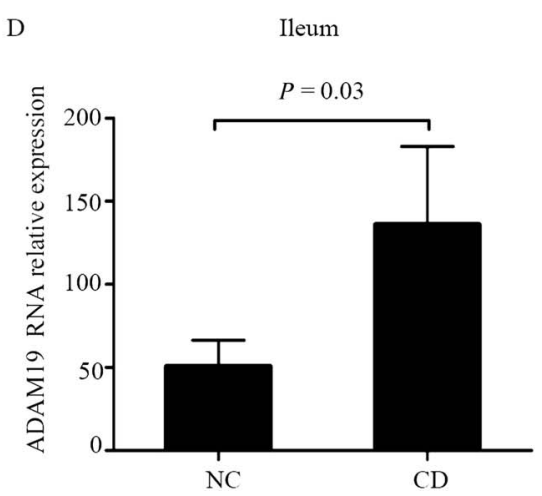

$10 \mathrm{mM}$ HEPES (pH 7.9), $10 \mathrm{mM} \mathrm{KCl}, 0.1 \mathrm{mM}$ EDTA, $0.2 \mathrm{mM}$ EGTA, and $0.5 \%$ Nonidet P40 (Sigma-Aldrich, Milan, Italy), supplemented with $1 \mathrm{mM}$ dithiothreitol, $10 \mathrm{mg} / \mathrm{mL}$ aprotinin, 10 $\mathrm{mg} / \mathrm{mL}$ leupeptin, $1 \mathrm{mM}$ phenylmethylsulfonyl fluoride, $1 \mathrm{mM}$ $\mathrm{Na}_{3} \mathrm{VO}_{4}$, and $1 \mathrm{mM} \mathrm{NaF}$. Lysates were clarified by centrifugation at $4^{\circ} \mathrm{C}, 12,000 \mathrm{~g}$ for 30 minutes, and separated on $8 \%$ sodium dodecyl sulfate-polyacrylamide gel electrophoresis. ADAM19 was detected using a rabbit anti-human ADAM19 (final dilution 1:110; Abgent, Inc., San Diego, CA) followed by a horseradish peroxidase-conjugated goat anti-rabbit IgG monoclonal antibody (final dilution 1:30,000; Dako, Milan, Italy). The reaction was detected with a sensitive-enhanced chemiluminescence kit (West PICO; Pierce, Rockford, IL). After the analysis of ADAM19, blots were stripped and incubated with a mouse anti-human $\beta$-actin antibody (final dilution 1:5000) as internal loading control, followed by a goat antimouse antibody conjugated to horseradish peroxidase (final dilution 1:20,000; Dako). ADAM19 was also evaluated in supernatants of DLD-1 cells and normal colonic mucosal explants cultured as described previously.

\section{Immunohistochemistry}

Immunohistochemistry was performed on archival, formalin-fixed paraffin-embedded colonic sections of 5 patients with UC, 4 patients with $\mathrm{CD}$, and $4 \mathrm{NC}$. Additional sections were prepared from samples taken from the inflamed and

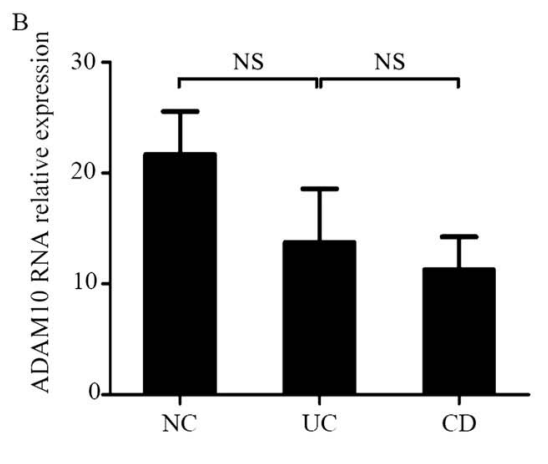


A

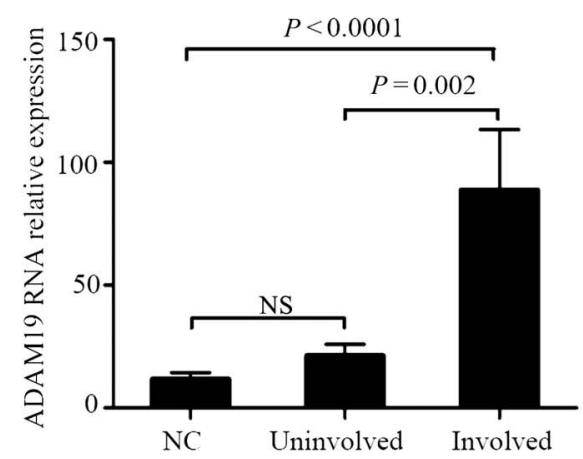

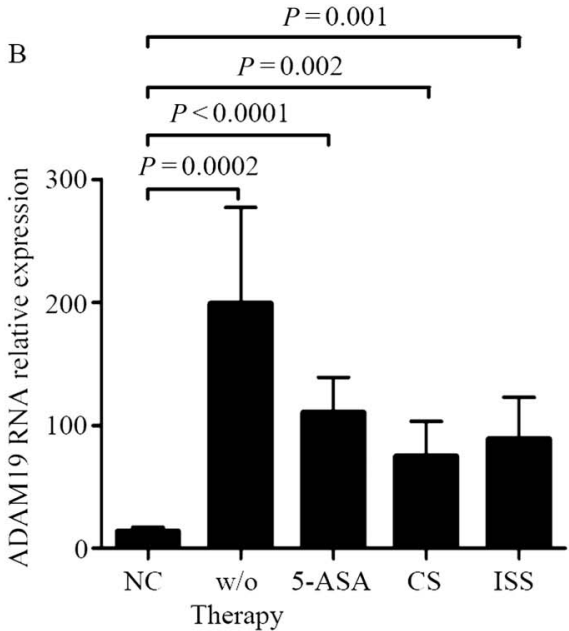

FIGURE 2. In IBD, ADAM19 RNA expression is upregulated in involved mucosa, and it is not influenced by current therapy. A, Paired biopsies taken from the involved and uninvolved mucosa of 8 patients with UC and 4 patients with CD and from 18 normal controls (NC) were analyzed for ADAM19 RNA expression by real-time PCR. Levels are normalized to $\beta$-actin, and data indicate mean \pm SEM of all samples. B, Colonic biopsies taken from $18 \mathrm{NC}, 10$ patients with IBD (6 CD and $4 \mathrm{UC}$ ) receiving no therapy (w/o therapy), 17 patients with IBD (5 CD and 12 UC) treated with mesalamine (5-ASA), 11 patients with IBD (4 CD and $7 \mathrm{UC}$ ) treated with steroids (CS), and 6 patients with IBD (4 CD and 2 UC) treated with immunomodulators (ISS) were analyzed for ADAM19 RNA expression by real-time PCR. Levels are normalized to $\beta$-actin. Data indicate mean \pm SEM of all samples. NS, not significant.

noninflamed areas of 3 patients with UC. The sections were deparaffinized and dehydrated through xylene and ethanol, and the antigen retrieval was performed in citrate buffer $(\mathrm{pH} 6.0)$ for 20 minutes in a microwave. Immunohistochemical staining was performed using a rabbit monoclonal antibody directed against human ADAM19 (final dilution 1:50) at room temperature for 1 hour followed by a biotin-free HRP-polymer detection technology with 3,3'diaminobenzidine (DAB) as a chromogen (MACH 4 Universal HRP-Polymer Kit; Biocare Medical, Concord, CA). The sections were counterstained with hematoxylin, dehydrated, and mounted. Isotype control IgG-stained sections were prepared under identical immunohistochemical conditions as described above, replacing the primary ADAM19 antibody with a purified rabbit normal IgG control antibody (R\&D Systems). The ADAM19expressing cells in both epithelial and lamina propria mononuclear compartments were counted by blinded pathologists using IAS 2000 System (Delta Sistemi, Rome, Italy) and expressed as number of cells per high-power field.

Immunohistochemistry was also performed on archival frozen sections of ileal mucosal samples taken from 3 patients with $\mathrm{CD}$ and $3 \mathrm{NC}$. Tissue sections were prepared as follows: samples were embedded in a cryostat-mounting medium (Neg50 Frozen Section Medium; Thermo Scientific, Langenselbold, Germany), snap frozen, and stored at $-80^{\circ} \mathrm{C}$ until sectioning. Samples were cut at $6 \mu \mathrm{m}$ thickness in a cryostat (Microm Cryostat HM 550; Thermo Scientific), mounted onto superfrost plus glass slides (Thermo Scientific), and fixed in $4 \%$ paraformaldehyde for 20 minutes. Tissue sections were incubated with a rabbit anti-human ADAM19 (final dilution 1:50) for 1 hour at room temperature, followed by a horseradish peroxidase-conjugated goat anti-rabbit IgG monoclonal antibody. Immunoreactive cells were visualized using DAB and lightly counterstained with hematoxylin. Isotype control sections were prepared under identical immunohistochemical conditions, replacing the primary antibody with a purified normal rabbit IgG control antibody (R\&D Systems).

\section{Immunofluorescence}

DLD-1 and HCT-116 cells were plated onto chamber slides $\left(1 \times 10^{4}\right.$ cells/well $)$ in RPMI 1640 medium supplemented with $10 \%$ fetal bovine serum and $\mathrm{P} / \mathrm{S} / \mathrm{G}$. The day after, cells were gently washed with phosphate-buffered saline and fixed with $4 \%$ paraformaldehyde for 20 minutes. Double immunofluorescence was performed as follows: cells were covered with Protein Block Serum-Free (Dako) and Streptavidin-Biotin blocking kit (Vector Laboratories, Inc., Burlingame, CA) to reduce nonspecific background staining; next, cells were incubated with a primary rat anti-human zonula occludens-1 (ZO-1) antibody (final dilution 1:100; BD Pharmingen, Heidelberg, Germany), and antigen detection was performed using TSA Fluorescein System (PerkinElmer, Rodgau, Germany). Cells were then incubated with a rabbit antihuman ADAM19 antibody (final dilution 1:50) overnight at $4^{\circ} \mathrm{C}$ followed by a Cy3-Donkey anti-Rabbit IgG $(\mathrm{H}+\mathrm{L})$ for 1 hour (final dilution 1:200; Jackson ImmunoResearch Laboratories, Inc., West Grove, PA). The nuclei were counterstained with Hoechst 3342 (Invitrogen). Isotype control slide were prepared under identical immunohistochemical conditions, replacing the primary antibodies with purified specific IgG control antibodies. Pictures were taken

504 | wwwibdjournal.org 
A
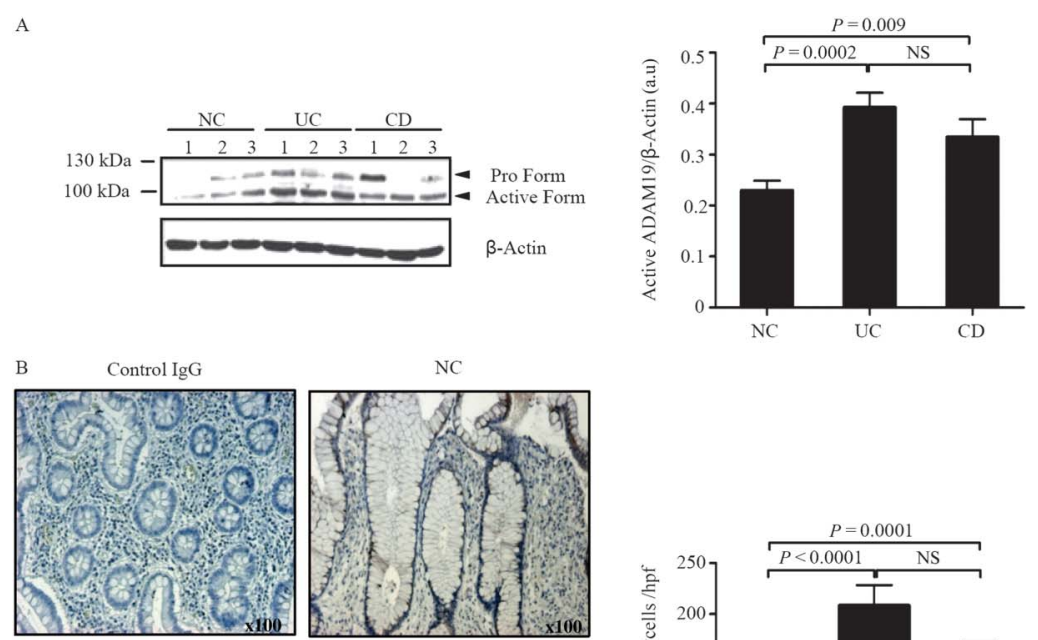

CD

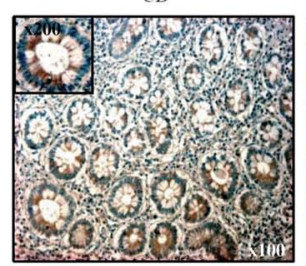

C
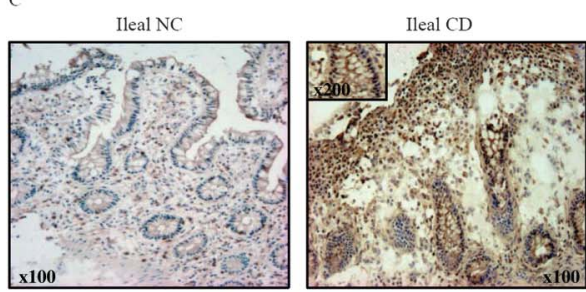

D

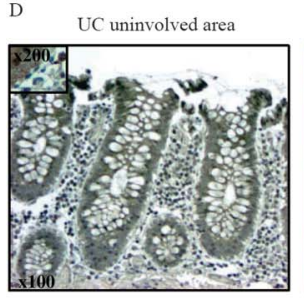

NC

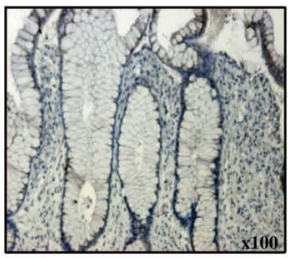

UC
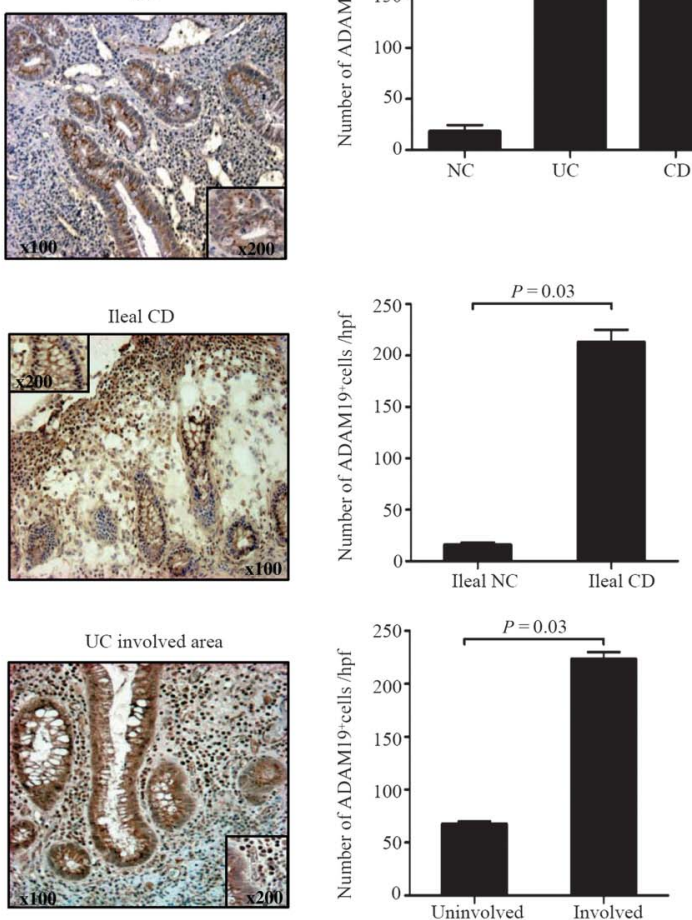

FIGURE 3. ADAM19 protein expression is increased in IBD. A, Representative Western blots showing ADAM19 and $\beta$-actin in total proteins extracted from mucosal samples of 3 normal controls (NC), 3 patients with UC, and 3 patients with CD. Right panel shows the quantitative analysis of active form of ADAM19/ $\beta$-actin ratio in mucosal samples taken from $9 \mathrm{NC}, 9$ patients with UC, and 9 patients with CD as measured by densitometry scanning of Western blots. Values are expressed in arbitrary units (a.u.) and indicate mean \pm SEM of all samples. NS, not significant. $B$, Representative photomicrographs ( $\times 100$ original magnification) of ADAM19-stained paraffin-embedded sections of surgical samples taken from $1 \mathrm{NC}, 1$ patient with UC, and 1 patient with CD. Isotype control antibody-stained section is also shown. In IBD, ADAM19-positive cells are evident in both the epithelial and lamina propria compartments. Higher magnification photomicrographs $(\times 200)$ are shown in the insets. Right panel shows the number of ADAM19-positive cells per high-power field (hpf) in colonic sections taken from 4 NC, 5 patients with UC, and 4 patients with CD. Data indicate mean \pm SEM. C, Representative photomicrographs (original magnification $\times 100$ ) showing ADAM19-positive cells in frozen sections of surgical ileal samples taken from $1 \mathrm{NC}$ and 1 patient with CD. Isotype control antibody-stained CD section is also shown. In IBD, ADAM19positive cells are evident in both the epithelial and lamina propria compartments. Inset shows higher magnification image $(\times 200)$. Right panel shows the number of ADAM19-positive cells per hpf in both the epithelial and lamina propria compartments of ileal sections taken from 3 NC and 3 patients with CD. Data indicate mean \pm SEM of all samples. D, Representative photomicrographs (original magnification $\times 100$ ) of ADAM19stained sections of colonic mucosal samples taken from involved and uninvolved mucosa of 1 patient with UC. Insets show higher magnification ( $\times 200)$ images. Right panel shows the number of ADAM19-positive cells per hpf in both the epithelial and lamina propria compartments of colonic sections taken from 3 patients with UC. Data indicate the mean \pm SEM of all samples. 
A

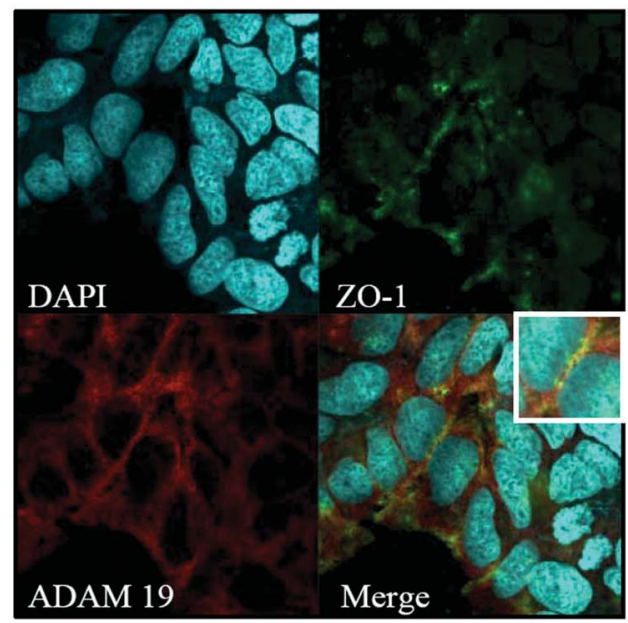

DLD-1

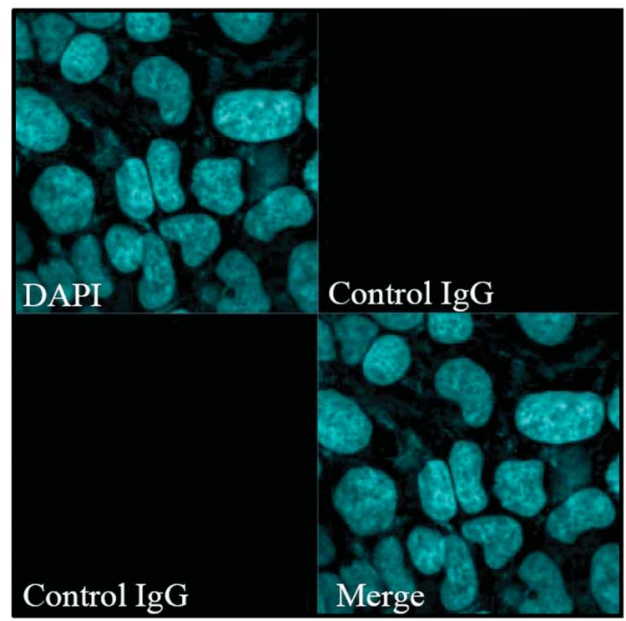

B

HCT 116
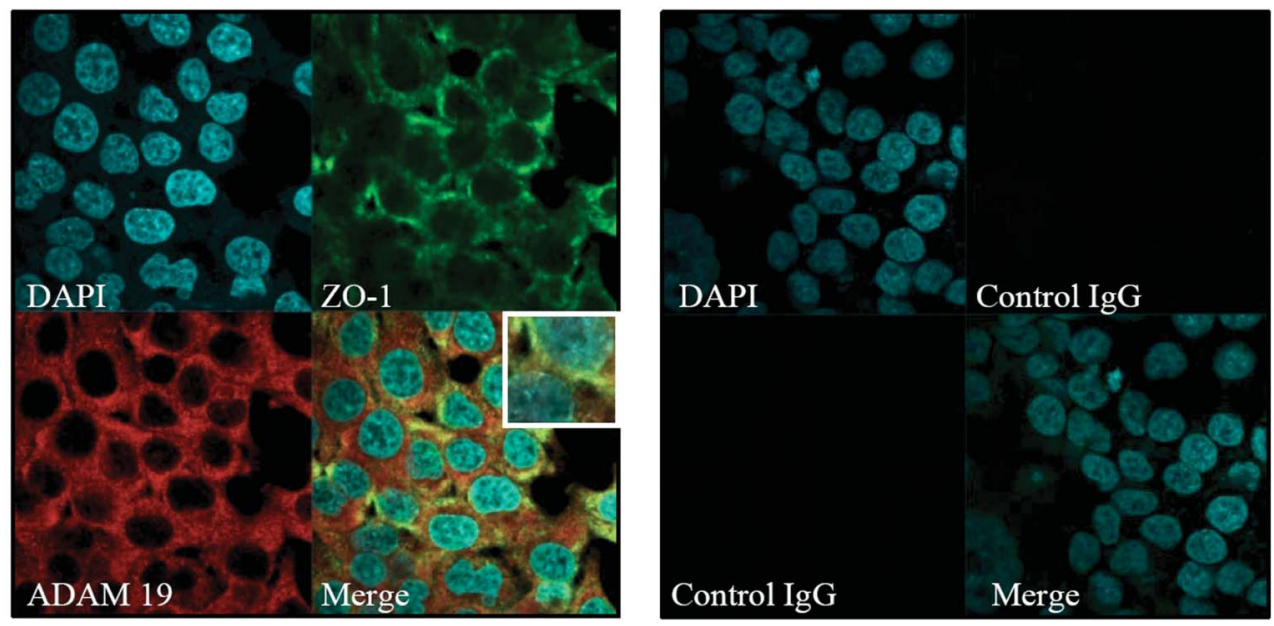

FIGURE 4. Colonic epithelial cells express ADAM19 on the plasmatic membrane. Representative images of confocal double-labeling immunofluorescence microscopy (original magnification $\times 400$ ) of DLD-1 (A) and HCT-116 (B) cells for ADAM19 (red) and ZO-1 (green). Merge (yellow) shows colocalization of ADAM19 and ZO-1 on the plasmatic membrane. Nuclei are stained with DAPI (blue). Staining with isotype control antibodies is also shown. Insets show higher magnification images $(\times 600)$.

using a confocal microscope (LSM TPMT; Carl Zeiss Microscopy, Jena, Germany).

\section{Statistical Analysis}

Differences between groups were compared using the paired Student's $t$ test, the Mann-Whitney U test, the Wilcoxon signed-rank test, and 1-way analysis of variance test.

\section{RESULTS}

\section{ADAM19 But Not ADAM9 and ADAM10 RNA Transcripts Are Upregulated in IBD}

To evaluate the expression of ADAM family members in the human gut, ADAM9, ADAM10, and ADAM19 RNA transcripts were assessed in colonic biopsies taken from patients with CD, patients with UC, and NC. RNA transcripts of ADAM9 and ADAM10 did not significantly differ between IBD and NC (Fig. 1A, B). In contrast, greater ADAM19 RNA expression was seen in UC compared with $\mathrm{CD}$ and normal samples (Fig. 1C), although ADAM19 transcripts were significantly higher in patients with CD compared with NC (Fig. 1C). ADAM19 RNA expression was also increased in patients with ileal CD compared with NC (Fig. 1D). In patients with IBD, upregulation of ADAM19 RNA expression (Fig. 2A) was restricted to the areas of active inflammation and not influenced by the current therapies (Fig. 2B). We also confirmed that ADAM17 was increased in the inflamed mucosa of patients with IBD compared with unaffected areas of the same patients and NC (see Fig. Supplemental Digital Content 1, http://links.lww.com/IBD/A36). 
A

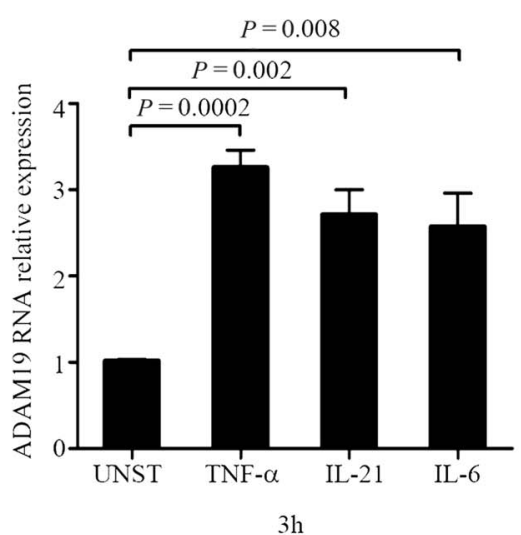

B

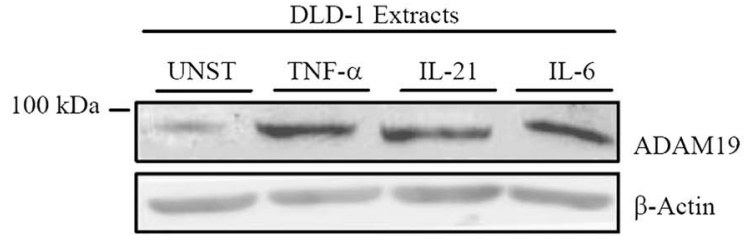

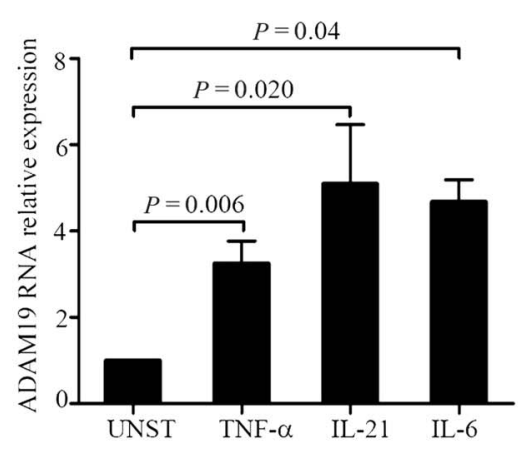

$6 \mathrm{~h}$

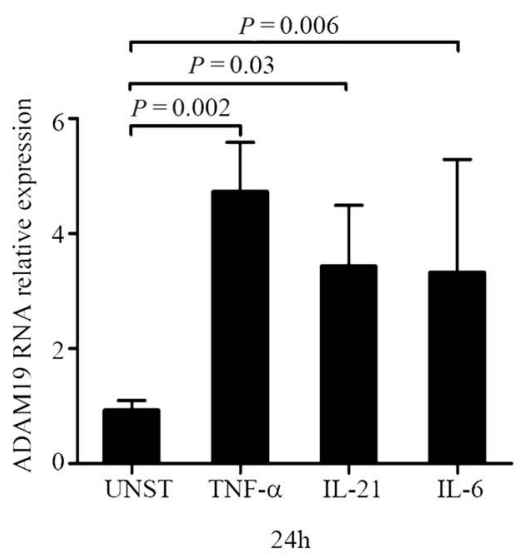

$24 \mathrm{~h}$

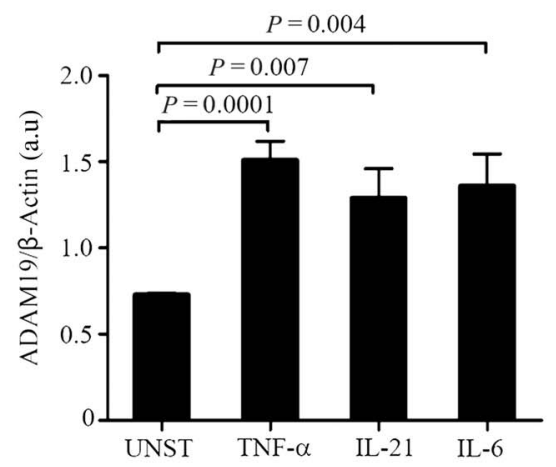

C

DLD-1 Supernatants

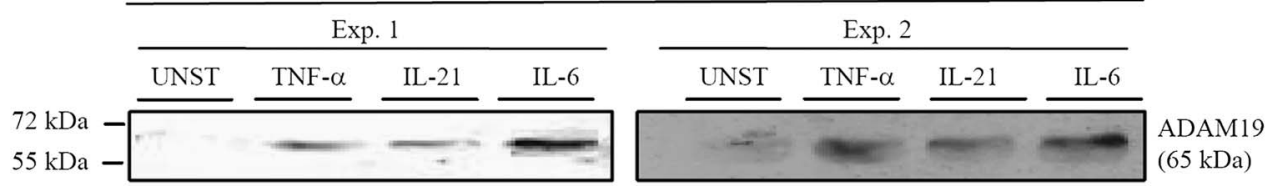

FIGURE 5. Inflammatory cytokines induce epithelial cells expression of ADAM19. A, DLD-1 cells were cultured with or without (unstimulated [UNST]), TNF- $\alpha(20 \mathrm{ng} / \mathrm{mL}), \mathrm{IL}-21(100 \mathrm{ng} / \mathrm{mL})$, and IL-6 $(20 \mathrm{ng} / \mathrm{mL})$ for the indicated time points, and ADAM19 RNA expression was analyzed by real-time PCR. Data indicate mean \pm SEM of 6 independent experiments. B, Representative Western blots showing ADAM19 and $\beta$-actin in total proteins extracted from DLD-1 cells treated as indicated above for 48 hours. Right panel shows the quantitative analysis of ADAM19/ $\beta$-actin ratio in DLD-1 cell protein extracts. Values are expressed in arbitrary units (a.u.) and indicate mean \pm SEM of 6 separate experiments. C, Representative Western blots showing the expression of ADAM19 soluble form in supernatants of DLD-1 cells cultured as indicated in (B). Two of 6 independent experiments in which similar results were obtained are shown.

\section{ADAM19 Protein Expression Is Increased in IBD Tissue}

Next, we examined ADAM19 protein expression in IBD and control samples by Western blotting and immunohistochemistry. Two bands corresponding to the precursor and active form of ADAM19 were evident in most control and CD samples and all UC samples (Fig. 3A). However, expression of the active form of ADAM19 was significantly increased in both UC and CD samples compared with controls (Fig. 3A). Immunohistochemical analysis confirmed that ADAM19-positive cells were more frequent in the inflamed areas of patients with IBD compared with controls (Fig. 3B). In particular, ADAM19 was mostly expressed by epithelial cells and, to a lesser extent, by lamina propria mononuclear cells in both $\mathrm{CD}$ and $\mathrm{UC}$ (Fig. 3B). ADAM19 protein expression was also upregulated in the inflamed ileum of patients with CD compared with NC (Fig. 3C). In line with the above data, high ADAM19 protein expression was seen in the inflamed mucosa of patients with UC compared with uninvolved uninflamed mucosa of the same patients (Fig. 3D).

\section{ADAM19 Expression Is Enhanced by Inflammatory Cytokines in a Gut Epithelial Cell Line and Tissue Explants}

Membrane localization of ADAM19 in epithelial cells was examined by immunofluorescence. ADAM19 colocalized with ZO-1, a tight junction-associated protein, in DLD-1 cells (Fig. 4A) and HCT-116 cells (Fig. 4B). The demonstration that ADAM19 is overexpressed in the inflamed tissue of UC and CD prompted us to examine whether it is positively regulated by some of the inflammatory cytokines produced in excess in both IBDs. ${ }^{1-3}$ Because epithelial cells are the major source of ADAM19 in IBD, we initially 

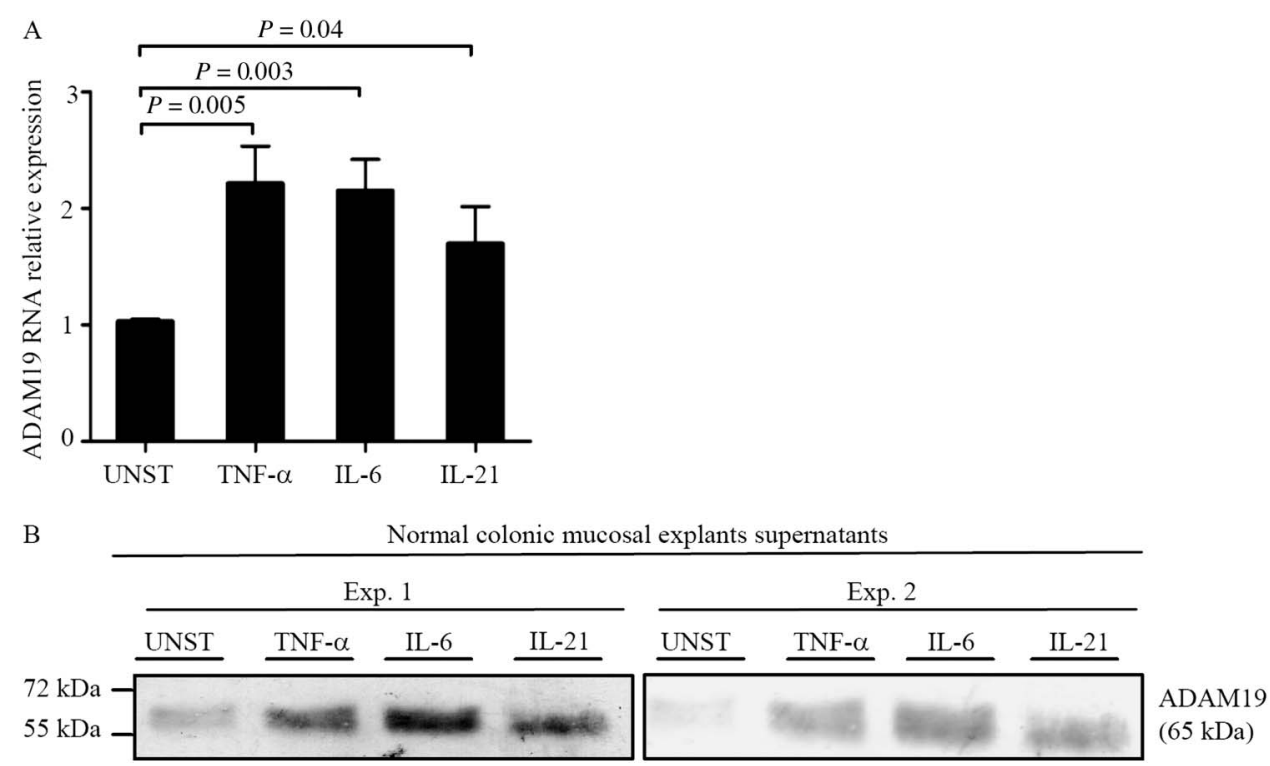

FIGURE 6. IBD-related inflammatory cytokines enhance ADAM19 production in normal colonic explants. A, Normal colonic mucosal explants were cultured with or without (unstimulated[UNST]), TNF- $\alpha(20 \mathrm{ng} / \mathrm{mL}), \mathrm{IL}-6(20 \mathrm{ng} / \mathrm{mL})$, and IL-21 (100 ng/mL) for 24 hours, and ADAM19 RNA expression was analyzed by real-time PCR. Data indicate mean \pm SEM of 4 experiments. B, Representative Western blots showing ADAM19 soluble form in supernatants of normal colonic mucosal explants cultured as described in (A) for 24 hours. Two of 4 independent experiments in which similar results were obtained are shown.

examined how ADAM19 is regulated in a colonic cancer cell line. ADAM19 RNA expression was upregulated in DLD-1 cells by TNF- $\alpha$, IL-6, and IL-21 (Fig. 5A). This effect was evident as early as 3 hours after the cytokine stimulation and persisted over the time (Fig. 5A). Western blotting experiments showed DLD-1 cells to constitutively express the active form of ADAM19 and to increase its production following stimulation with TNF- $\alpha$, IL-6, and IL-21 (Fig. 5B). A smaller fragment of approximately $65 \mathrm{kDa}$ and known to be the processed N-terminal fragment of soluble human ADAM19 ${ }^{28}$ was evident in DLD-1 supernatants, especially in response to TNF- $\alpha$, IL-6, and IL-21 (Fig. 5C).

To extend our observations to primary cells, we stimulated normal colonic explants with TNF- $\alpha$, IL-6, and IL-21. Consistent with the in vitro data, each of these cytokines enhanced ADAM19 RNA expression (Fig. 6A) and the quantity of secreted ADAM19 protein in the supernatants (Fig. 6B).

\section{Anti-TNF- $\alpha$ Inhibits ADAM19 Expression in IBD}

In a final set of experiments, we evaluated whether ADAM19 was downregulated by anti-TNF- $\alpha$. We initially assessed ADAM19 expression in colonic biopsies taken from patients with IBD before and after a successful treatment with IFX. Immunohistochemistry analysis showed that IFX markedly reduced the percentage of ADAM19-positive cells in both epithelial and lamina propria compartments (Fig. 7A). ADAM19 expression was also evaluated in ex vivo mucosal explants taken from patients with IBD cultured in the presence of IFX or control IgG for 24 hours. Notably, ADAM19 RNA transcripts were significantly downregulated by IFX (Fig. 7B).
To examine whether anti-TNF- $\alpha$ treatment directly modulates ADAM19, we examined ADAM19 RNA transcripts in DLD-1 cells treated with IFX. IFX reduced ADAM19 expression (see Fig. A, Supplemental Digital Content 2, http://links.lww.com/IBD/A37). In contrast, no significant changes in ADAM17 RNA transcripts were seen after the IFX treatment (see Fig. B, Supplemental Digital Content 2, http://inks.lww.com/IBD/A37). Additionally, the IFX treatment did not alter the fraction of $\mathrm{AV}+$ and/or PI+ DLD-1 cells (see Fig. C, Supplemental Digital Content 2, http://links.lww.com/IBD/A37).

\section{DISCUSSION}

TNF- $\alpha$ is a proinflammatory cytokine that plays a key role in immune-mediated diseases, such as $\mathrm{CD}$ and UC. High TNF- $\alpha$ expression is seen in the inflamed gut of patients with IBD, and neutralization of the cytokine is associated with clinical benefit and resolution of the mucosal inflammation. ${ }^{29,30}$ After its synthesis, TNF- $\alpha$ undergoes proteolytic release from the plasma membrane, a tightly controlled phenomenon, which is termed "protein ectodomain shedding." Shedding of transmembranebound TNF- $\alpha$ is mainly mediated by ADAM17, an enzyme that is highly expressed in IBD tissue. ${ }^{20-23}$ However, it has been reported that other ADAMs (e.g., ADAM9, ADAM10, and ADAM19) are important sheddases of TNF- $\alpha{ }^{22}$

This study was designed to investigate the expression and distribution of ADAM9, ADAM10, and ADAM19 in the gut of patients with IBD and to determine factors involved in their regulation. We observed no significant difference in ADAM9 and ADAM10 RNA expression between NC and patients with IBD. In 
A

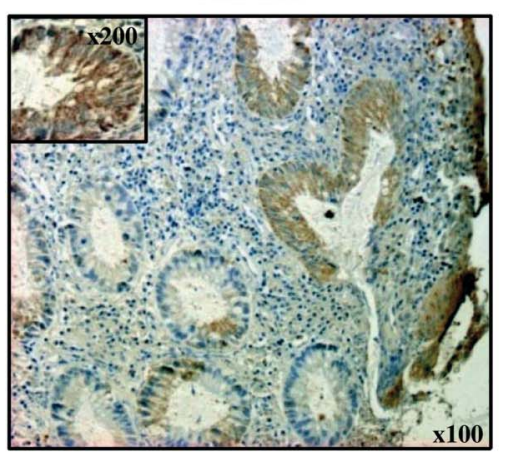

PRE-IFX

Control IgG

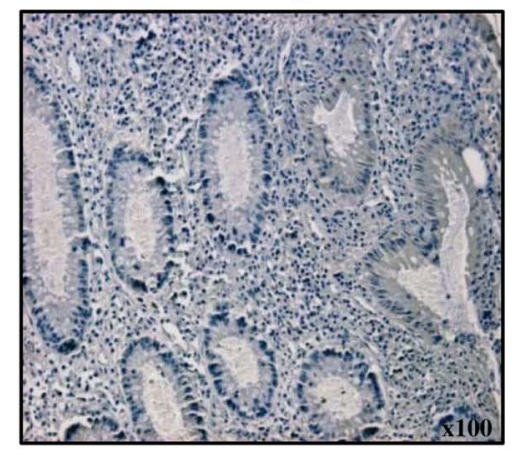

POST-IFX
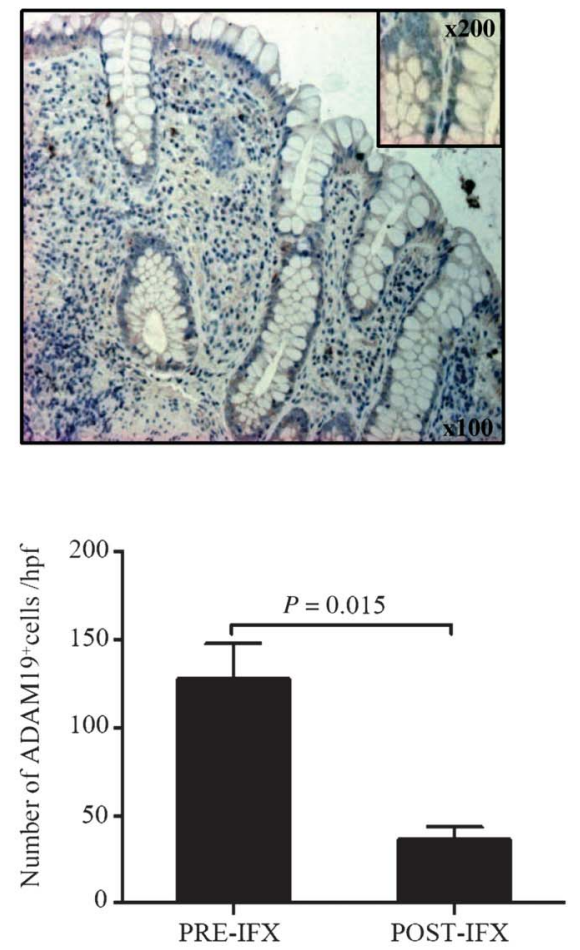

B IBD explants

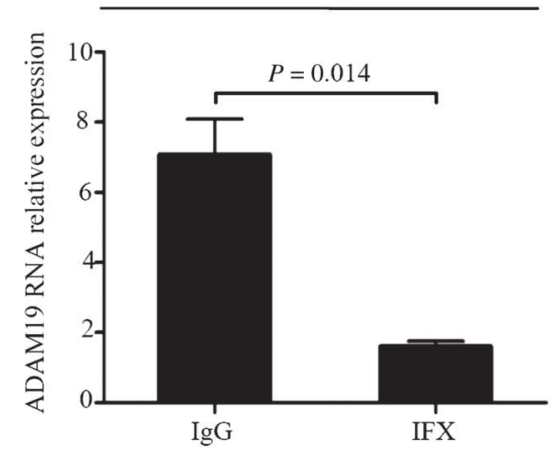

FIGURE 7. Infliximab downregulates ADAM19 expression in IBD. A, Representative photomicrographs (original magnification $\times 100$ ) of ADAM19stained sections of 1 patient with CD, before and after infliximab (IFX) therapy. Isotype control antibody-stained section is also shown. Insets show higher magnification ( $\times 200)$ images. Right bottom histogram shows the number of ADAM19-positive cells per high-power field (hpf) in colonic sections taken from 2 patients with UC and 3 patients with CD before and after the IFX treatment. Data indicate mean \pm SD of all samples. $\mathrm{B}$, Colonic mucosal explants taken from 4 patients with CD were cultured with infliximab (IFX) or control IgG for 24 hours. ADAM19 RNA expression was then measured by real-time PCR. Date indicate mean \pm SEM of all experiments.

contrast, ADAM19 transcripts were markedly upregulated in patients with UC and, to a lesser extent, in patients with $\mathrm{CD}$. The reason why ADAM19 expression is more pronounced in $\mathrm{UC}$ is not known, even though the severity of the mucosal inflammation and/ or factors locally produced could contribute to differently modulate ADAM19 in IBD. The increased ADAM19 expression, both at RNA and at protein levels, was restricted to mucosal areas with active inflammation. Additionally, the expression of this sheddase in samples taken from the uninflamed mucosa of patients with IBD did not differ from that seen in the colon of controls. Moreover,
ADAM19 expression did not differ between patients with IBD taking no therapy and those who were receiving drugs.

Western blotting and immunohistochemistry analysis showed that ADAM19 protein was abundantly expressed in IBD, particularly in epithelial cells and in mononuclear cells in the lamina propria. Although we have very little understanding of the control of ADAM19 in the human gut, our data suggest that cytokines overproduced in the inflamed tissue of patients with IBD may contribute to enhance ADAM19 expression. Indeed, stimulation of both epithelial cell lines and normal colon tissue 
explants with TNF- $\alpha$, IL-6, and IL-21 increased ADAM19 expression, and treatment of IBD explants with IFX reduced ADAM19 gene expression. This latter finding is in line with the demonstration that patients with IBD responding to the IFX treatment exhibited a marked colonic reduction of ADAM19 expression and that IFX reduced ADAM19 RNA transcripts in DLD-1. Although these data suggest that IFX may directly modulate ADAM19 expression, we do not exclude the possibility that the decreased ADAM19 seen in patients following the IFX treatment can also rely on resolution of the inflammatory process.

The activity of ADAM19 is regulated at multiple levels, including transcription, translation, and release from the cell membrane. ${ }^{28,31,32}$ The protein has multiple domains, including signal peptide, a pro-domain, a MMP domain, a disintegrin domain, a cysteinerich domain, an epidermal growth factor-like domain, a transmembrane domain, and a cytoplasmic domain. ${ }^{33,34}$ Pro-protein-converting enzymes such as furin remove the pro-domain and activate the MMP domain, thus producing the active form ADAM19 following autolysis of the cysteine-rich domain. ${ }^{32}$ Cellular processing and activation of ADAM19 is enhanced by lipopolysaccharide and phorbol 12-myristate 13-acetate, a protein kinase $\mathrm{C}$ activator, whereas calmodulin antagonists impair the cleavage. ${ }^{28,35}$ Our study expands on this information and shows that inflammatory cytokines can enhance transcription and production of ADAM19. There are no commercially available reagents to characterize the activity of human ADAM19. Therefore, further studies will be necessary to ascertain whether, in IBD, high ADAM19 expression correlates with enhanced activity of the protein.

In contrast to other members of the metzincin superfamily of zinc MMPs, ADAM19 does not cleave typical MMP substrates, such as type 1 collagen, gelatin, and casein, and its activity is not inhibitable by TIMP 1 and TIMP $2 .{ }^{30}$ Other than promoting TNF- $\alpha$ shedding, ADAM19 is involved in the proteolytic cleavage of members of the EGFR family, such as heparin-binding EGF and neuregulin, and in the extravasation of monocytes. ${ }^{36-38}$ Indeed, it is highly likely that the increased ADAM19 on epithelial cells in IBD can associate with its role as a sheddase for these other molecules because epithelial production of TNF- $\alpha$ is minor compared with that of monocytes/ macrophages in IBD.

ADAM19 is also abundantly expressed in many immuneinflammatory renal pathologies, where it is associated with glomerular damage, chronic allograft nephropathy, inflammatory lung disorders, and myopathies. ${ }^{39-43}$ ADAM19 is also upregulated in human brain tumors such as astrocytoma and glioblastoma and correlates with the invasiveness of glioma. ${ }^{44}$ It is also overexpressed by many human cancerous cell lines, including cancers of the colon, ovary, lung, and brain. ${ }^{31}$ In general, these findings suggest that targeted inhibition of ADAM19 may be crucial for the treatment of certain types of tumors and inflammatory diseases.

In conclusion, our findings show that ADAM19 is overexpressed in the inflamed intestine of patients with IBD and that production of this protein can be enhanced by inflammatory cytokines. Because ADAM19 is involved in the shedding of membrane-bound TNF- $\alpha$, along with ADAM17, it suggests that this enzyme may play a key role in the pathogenesis of IBD. However, the fact that we were not able to silence ADAM19 in our cell systems using commercial small interfering RNA leaves open the possibility that ADAM19 can also contribute to maintain the epithelial barrier integrity and/or induce protective proteins.

\section{REFERENCES}

1. Abraham C, Cho JH. Inflammatory bowel disease. N Engl J Med. 2009; 361:2066-2078.

2. Bouma G, Strober W. The immunological and genetic basis of inflammatory bowel disease. Nat Rev Immunol. 2003;3:521-533.

3. Strober W, Fuss I, Mannon P. The fundamental basis of inflammatory bowel disease. J Clin Invest. 2007;117:514-521.

4. Wajant H, Pfizenmaier K, Scheurich P. Tumor necrosis factor signaling. Cell Death Differ. 2003;10:45-65.

5. Vilcek J, Lee TH. Tumor necrosis factor. New insights into the molecular mechanisms of its multiple actions. J Biol Chem. 1991;266:7313-7316.

6. Liu ZG. Molecular mechanism of TNF signaling and beyond. Cell Res. 2005; 15:24-27.

7. Rutgeerts P, D'Haens G, Targan S, et al. Efficacy and safety of retreatment with anti-tumor necrosis factor antibody (infliximab) to maintain remission in Crohn's disease. Gastroenterology. 1999;117:761-769.

8. Hanauer SB, Feagan BG, Lichtenstein GR, et al. Maintenance infliximab for Crohn's disease: the ACCENT I randomised trial. Lancet. 2002;359: 1541-1549.

9. Rutgeerts P, Sandborn WJ, Feagan BG, et al. Infliximab for induction and maintenance therapy for ulcerative colitis. $N$ Engl J Med. 2005;353: $2462-2476$.

10. Colombel JF, Sandborn WJ, Rutgeerts P, et al. Adalimumab for maintenance of clinical response and remission in patients with Crohn's disease: the CHARM trial. Gastroenterology. 2007;132:52-65.

11. Targan SR, Hanauer SB, van Deventer SJ, et al. A short-term study of chimeric monoclonal antibody cA2 to tumor necrosis factor alpha for Crohn's disease. Crohn's Disease cA2 Study Group. $N$ Engl J Med. 1997;337:1029-1035.

12. Rutgeerts P, Vermeire S, Van Assche G. Biological therapies for inflammatory bowel diseases. Gastroenterology. 2009;136:1182-1197.

13. Gisbert JP, Panes J. Loss of response and requirement of infliximab dose intensification in Crohn's disease: a review. Am J Gastroenterol. 2009; 104:760-767.

14. Fidder H, Schnitzler F, Ferrante M, et al. Long-term safety of infliximab for the treatment of inflammatory bowel disease: a single-centre cohort study. Gut. 2009;58:501-508.

15. Nunez Martinez O, Ripoll Noiseux C, Carneros Martin JA, et al. Reactivation tuberculosis in a patient with anti-TNF-alpha treatment. $\mathrm{Am} \mathrm{J}$ Gastroenterol. 2001;96:1665-1666.

16. Schneeweiss S, Korzenik J, Solomon DH, et al. Infliximab and other immunomodulating drugs in patients with inflammatory bowel disease and the risk of serious bacterial infections. Aliment Pharmacol Ther. 2009;30:253-264.

17. Hanauer SB, Sandborn WJ, Rutgeerts P, et al. Human anti-tumor necrosis factor monoclonal antibody (adalimumab) in Crohn's disease: the CLASSIC-I trial. Gastroenterology. 2006;130:323-333; quiz 591.

18. Spriggs DR, Deutsch S, Kufe DW. Genomic structure, induction, and production of TNF-alpha. Immunol Ser. 1992;56:3-34.

19. Kriegler M, Perez C, DeFay K, et al. A novel form of TNF/cachectin is a cell surface cytotoxic transmembrane protein: ramifications for the complex physiology of TNF. Cell. 1988;53:45-53.

20. McGeehan GM, Becherer JD, Bast RC Jr, et al. Regulation of tumour necrosis factor-alpha processing by a metalloproteinase inhibitor. Nature. 1994;370:558-561.

21. Moss ML, Jin SL, Milla ME, et al. Cloning of a disintegrin metalloproteinase that processes precursor tumour-necrosis factor-alpha. Nature. 1997;385:733-736.

22. Brynskov J, Foegh P, Pedersen G, et al. Tumour necrosis factor alpha converting enzyme (TACE) activity in the colonic mucosa of patients with inflammatory bowel disease. Gut. 2002;51:37-43. 
23. Colon AL, Menchen LA, Hurtado O, et al. Implication of TNF-alpha convertase (TACE/ADAM17) in inducible nitric oxide synthase expression and inflammation in an experimental model of colitis. Cytokine. 2001;16:220-226.

24. Zheng Y, Saftig P, Hartmann D, et al. Evaluation of the contribution of different ADAMs to tumor necrosis factor alpha (TNFalpha) shedding and of the function of the TNFalpha ectodomain in ensuring selective stimulated shedding by the TNFalpha convertase (TACE/ADAM17). J Biol Chem. 2004;279:42898-42906.

25. Daperno M, D'Haens G, Van Assche G, et al. Development and validation of a new, simplified endoscopic activity score for Crohn's disease: the SES-CD. Gastrointest Endosc. 2004;60:505-512.

26. Schroeder KW, Tremaine WJ, Ilstrup DM. Coated oral 5-aminosalicylic acid therapy for mildly to moderately active ulcerative colitis. A randomized study. N Engl J Med. 1987;317:1625-1629.

27. Monteleone G, Kumberova A, Croft NM, et al. Blocking Smad7 restores TGF-beta1 signaling in chronic inflammatory bowel disease. J Clin Invest. 2001;108:601-609.

28. Kang T, Park HI, Suh Y, et al. Autolytic processing at Glu586-Ser587 within the cysteine-rich domain of human adamalysin 19/disintegrinmetalloproteinase 19 is necessary for its proteolytic activity. J Biol Chem. 2002;277:48514-48522.

29. Reinecker HC, Steffen M, Witthoeft T, et al. Enhanced secretion of tumour necrosis factor-alpha, IL-6, and IL-1 beta by isolated lamina propria mononuclear cells from patients with ulcerative colitis and Crohn's disease. Clin Exp Immunol. 1993;94:174-181.

30. Sandborn WJ, Hanauer SB. Antitumor necrosis factor therapy for inflammatory bowel disease: a review of agents, pharmacology, clinical results, and safety. Inflamm Bowel Dis. 1999;5:119-133.

31. Qi B, Newcomer RG, Sang QX. ADAM19/adamalysin 19 structure, function, and role as a putative target in tumors and inflammatory diseases. Curr Pharm Des. 2009;15:2336-2348.

32. Kang T, Zhao YG, Pei D, et al. Intracellular activation of human adamalysin 19 /disintegrin and metalloproteinase 19 by furin occurs via one of the two consecutive recognition sites. J Biol Chem. 2002; 277:25583-25591.
33. Kurohara K, Matsuda Y, Nagabukuro A, et al. Meltrin beta (ADAM19) gene: cloning, mapping, and analysis of the regulatory region. Biochem Biophys Res Commun. 2000;270:522-527.

34. Wei P, Zhao YG, Zhuang L, et al. Expression and enzymatic activity of human disintegrin and metalloproteinase ADAM19/meltrin beta. Biochem Biophys Res Commun. 2001;280:744-755.

35. Tanabe C, Hotoda N, Sasagawa N, et al. ADAM19 autolysis is activated by LPS and promotes non-classical secretion of cysteine-rich protein 2 . Biochem Biophys Res Commun. 2010;396:927-932.

36. Horiuchi K, Zhou HM, Kelly K, et al. Evaluation of the contributions of ADAMs 9, 12, 15, 17, and 19 to heart development and ectodomain shedding of neuregulins beta1 and beta2. Dev Biol. 2005;283:459-471.

37. Shirakabe K, Wakatsuki S, Kurisaki T, et al. Roles of Meltrin beta /ADAM19 in the processing of neuregulin. J Biol Chem. 2001;276:9352-9358.

38. Fritsche J, Muller A, Hausmann M, et al. Inverse regulation of the ADAM-family members, decysin and MADDAM/ADAM19 during monocyte differentiation. Immunology. 2003;110:450-457.

39. Melenhorst WB, van den Heuvel MC, Timmer A, et al. ADAM19 expression in human nephrogenesis and renal disease: associations with clinical and structural deterioration. Kidney Int. 2006;70:1269-1278.

40. Melenhorst WB, van den Heuvel MC, Stegeman CA, et al. Upregulation of ADAM19 in chronic allograft nephropathy. Am J Transplant. 2006;6: 1673-1681.

41. Roemer A, Schwettmann L, Jung M, et al. Increased mRNA expression of ADAMs in renal cell carcinoma and their association with clinical outcome. Oncol Rep. 2004;11:529-536.

42. Dijkstra A, Postma DS, Noordhoek JA, et al. Expression of ADAMs ("a disintegrin and metalloprotease") in the human lung. Virchows Arch. 2009; 454:441-449.

43. Dehmel T, Janke A, Hartung HP, et al. The cell-specific expression of metalloproteinase-disintegrins (ADAMs) in inflammatory myopathies. Neurobiol Dis. 2007;25:665-674.

44. Wildeboer D, Naus S, Amy Sang QX, et al. Metalloproteinase disintegrins ADAM8 and ADAM19 are highly regulated in human primary brain tumors and their expression levels and activities are associated with invasiveness. J Neuropathol Exp Neurol. 2006;65:516-527. 